\title{
MULTICOMPONENT MODELS IN NUCLEAR ASTROPHYSICS
}

\author{
BERNARD DUCOMET \\ $C E A / D A M / D I F F$ \\ Département de Physique Théorique et Appliquée \\ F-91680 Bruyères le Châtel, France \\ E-mail: bernard.ducomet@cea.fr
}

\begin{abstract}
We consider hydrodynamical models describing the evolution of a gaseous star in which the presence of thermonuclear reactions between several species leads to a multicomponent formulation. In the case of binary mixtures, recent $3 \mathrm{D}$ results are evoked. In the one-dimensional situation, we can prove global estimates and stabilization for some simplified model.
\end{abstract}

1. Introduction. Stars can generally be considered as continuous media for which hydrodynamical description is natural, then the equations governing the evolution of stellar structure are almost identical to those describing ordinary fluid mechanics [30] [38] [18], except the fact that, due to various chemical reactions taking place in the medium (more precisely thermonuclear reactions), one has to consider a multicomponent mixture containing several particle species.

Multifluids have been the matter of a number of works in the past which, for our purposes, may be very roughly divided into those concerning multiphase flows and those involving multicomponent flows. The first kind of models suppose that, given several fluids, only one of them is present into each given ("bubble" like) macroscopic region of space, each bubble being plugged into an underlying fluid region. One is then faced with a multifluid free boundary problem [36] [39] for each subregion (see [50] [46] [15] [31] [32] for related mathematical results).

In the multicomponent formulation [17] [33], each component is simultaneously present at any point of the space and global hydrodynamical variables for the mixture may be identified: the density, momentum, internal energy and entropy corresponding to a "mean flow" with suitable state functions, then dynamics is coupled to a reaction-diffusion system describing the evolution of the individual densities with source terms telling how

2000 Mathematics Subject Classification: Primary 76N10; Secondary 80A32.

Key words and phrases: multicomponent flow, binary mixture, gaseous star.

The paper is in final form and no version of it will be published elsewhere. 
each species is produced (or destroyed) through chemical or thermonuclear reactions. Moreover the state functions depend now also on the individual concentrations. In the astrophysical setting, such reaction-diffusion systems lead to the chemical composition of stars [30].

Although multiphase flows may also be encountered in some astrophysical applications (models of plasma turbulence in stellar atmospheres [42]) we focus here on multicomponent flows involved in fundamental problems [38] [30] [44] as stellar evolution, nucleosynthesis or flows in interstellar medium which are presently actively studied by physicists. As an example of thermonuclear coupling in stars, let us only mention the so-called "PP II cycle" (Proton-Proton) describing describing the Helium chain in stellar nucleosynthesis [38] [30] [2]:

$$
\left\{\begin{array}{c}
{ }^{3} \mathrm{He}+{ }^{4} \mathrm{He} \rightarrow{ }^{7} \mathrm{Be}+\gamma, \\
{ }^{7} \mathrm{Be}+e^{-} \rightarrow{ }^{7} \mathrm{Li}+\nu, \\
{ }^{7} \mathrm{Li}+\mathrm{H} \rightarrow{ }^{8} \mathrm{Be}+\gamma, \\
{ }^{8} \mathrm{Be} \rightarrow 2^{4} \mathrm{He}+\gamma
\end{array}\right.
$$

where $H e$ is helium, $B e$ beryllium, $L i$ lithium, $H$ hydrogen, $e^{-}$an electron, $\nu$ a neutrino and $\gamma$ a photon. In fact, a huge number of such cycles (leading from the lighter to the heavyier nuclei) are present in stars and the competition between chemistry and gravitation is the core of stellar evolution.

In the following, we provide (section 2) a quick derivation of the multicomponent formulation, then (section 3) we present recent results of global existence for a simple model for binary mixtures finally we give in section 4 a stabilization result in the monodimensional case.

2. A general model. Let us start with a few words about multifluid systems, the description of which depends on the level of approximation one desires to assume.

In fact, the more precise way to deal with multifluids relies on kinetic theory [11] [8] leading to a complex Boltzmann formulation for the various distribution functions involved [7] [6].

This general framework allows to treat very out-of-equilibrium situations however, as we are interested in stellar evolution applications [30] [38], we restrict our study to the fluid regime for a mixture of $N$ reacting species.

In fact, even the complete multicomponent fluid system evoked above is still too complex to lead to a complete analysis. As it modelizes various physical situations going from reacting astrophysical plasmas to chemical reactors or superfluids, it is natural to specialize it to the relevent physics involved. A major simplification takes place when the dynamics can be reduced to a one-fluid main (mean) flow, with source-flux couplings to the individual species. This indeed is the case in the reactive fluids case where a (possibly complex) chemical kinetics is coupled to the main flow, but this procedure requires some further modelling to get the state functions and the various fluxes. 
Let us consider a mixture of $N$ Newtonian fluids, each of which is characterized by the variables $\rho_{i}, \mathbf{u}_{i}, \theta_{i}$ with stress tensor $\sigma_{i}=-p_{i} \mathbf{I}+\pi_{i} \mathbf{u}_{i}$, where $p_{i}\left(\rho_{i}, \theta_{i}\right)$ is the pressure and $\pi_{i}\left(\mathbf{u}_{i}\right)$ isthe viscous stress. We also suppose that a force $\mathbf{f}_{i}$ acts at each point in $i$.

We define the global density of the mixture as

$$
\rho:=\sum_{i=1}^{N} \rho_{i}
$$

and the global velocity as

$$
\rho \mathbf{u}:=\sum_{i=1}^{N} \rho_{i} \mathbf{u}_{i}
$$

If we sum the partial continuity equations, we get first the familiar conservation law

$$
\partial_{t} \rho+\nabla \cdot(\rho \mathbf{u})=0 .
$$

Now if we sum up the momentum equations and use the identity

$$
\sum_{i=1}^{N} \rho_{i} \mathbf{u}_{i} \otimes \mathbf{u}_{i}=\rho \mathbf{u} \otimes \mathbf{u}+\sum_{i=1}^{N} \rho_{i}\left(\mathbf{u}-\mathbf{u}_{i}\right) \otimes\left(\mathbf{u}-\mathbf{u}_{i}\right),
$$

we get formally a momentum-like conservation law

$$
\partial_{t}(\rho \mathbf{u})+\nabla \cdot(\rho \mathbf{u} \otimes \mathbf{u})=\nabla \cdot \sigma+\mathbf{f},
$$

where $\mathbf{f}:=\sum_{i=1}^{N} \mathbf{f}_{i}$.

Now the effective stress tensor is

$$
\sigma=\sum_{i=1}^{N}\left\{\sigma_{i}+\rho_{i}\left(\mathbf{u}-\mathbf{u}_{i}\right) \otimes\left(\mathbf{u}-\mathbf{u}_{i}\right)\right\}=\sum_{i=1}^{N}\left\{-p_{i}\left(\rho_{i}, \theta_{i}\right) \mathbf{I}+\pi_{i}+\rho_{i}\left(\mathbf{u}-\mathbf{u}_{i}\right) \otimes\left(\mathbf{u}-\mathbf{u}_{i}\right)\right\} .
$$

Then, in order to obtain a mean flow system we can absorb the $\left(\mathbf{u}-\mathbf{u}_{i}\right)$-quadratic term in this relation and make strong hypotheses on the partial stresses $\sigma_{i}$.

In the particular case of a one-temperature model and if all the species are perfect gas with the same constant $R_{i}=R$ i.e. $p_{i}\left(\rho_{i}, \theta_{i}\right)=R \rho_{i} \theta$, we get an effective pressure by applying the Dalton's law: the total pressure is the sum of the partial pressures

$$
p(\rho, \theta):=\sum_{i=1}^{N} p_{i}\left(\rho_{i}, \theta\right),
$$

however the viscous contribution

$$
\pi:=\sum_{i=1}^{N} \pi_{i}\left(\mathbf{u}_{i}\right),
$$

cannot in general be expressed as a functional $\pi(\mathbf{u})$.

The same arguments applied to the energy equation lead to similar difficulties.

A way out is to discard the $\mathbf{u}_{i}$ as dynamical variables and to define them by constitutive laws (a simple example of such is the Fick's diffusion law) in term of the temperature and the partial concentrations $c_{i}$, which amounts to suppose that although the species may diffuse in the mixture, they are dynamically advected by the mean flow only. 
2.1. The equations for a multicomponent reacting mixture. In this section we adopt the notations of Giovangigli [34].

In the following model, we suppose that all the species are mutually in local thermodynamical equilibrium, including the radiative contribution. Then the problem involves a unique temperature field for all the species. We also suppose that the mixture is driven by a mean flow described by the macroscopic variables $(\rho, \mathbf{u}, \theta)$

We consider the mixture as a viscous compressible fluid with no source of mass, the the mass conservation of individual species reads

$$
\partial_{t}\left(\rho Y_{i}\right)+\nabla \cdot\left(\rho Y_{i} \mathbf{u}\right)=w_{i}-\nabla \cdot \mathbf{J}_{i},
$$

for $i=1 \ldots N$, for the species mass fraction $Y_{i}=\frac{\rho}{\rho_{i}}$, where $\mathbf{J}_{i}$ is the diffusion flux of the species $i$

$$
\mathbf{J}_{i}=\rho Y_{i} \mathbf{v}_{i},
$$

where $\mathbf{v}_{i}$ is the diffusion velocity of the species $i$, and $w_{i}$ is the molar production rate for species $i$.

The species mass constraint (no global matter source) implies

$$
\sum_{i=1}^{N} \mathbf{J}_{i}=0, \text { and } \sum_{i=1}^{N} w_{i}=0,
$$

from which one gets the global mass conservation

$$
\partial_{t} \rho+\nabla \cdot(\rho \mathbf{u})=0 .
$$

Momentum conservation reads

$$
\partial_{t}(\rho \mathbf{u})+\nabla \cdot(\rho \mathbf{u} \otimes \mathbf{u})=\nabla \cdot \sigma+\rho \sum_{i=1}^{N} Y_{i} \mathbf{f}_{i}
$$

with the species mass fraction $Y_{i}=\frac{\rho}{\rho_{i}}$. Energy conservation reads

$$
\partial_{t}(\rho e)+\nabla \cdot(\rho e \mathbf{u})=\sigma: \nabla \mathbf{u}-\nabla \cdot \mathbf{q}+\sum_{i=1}^{N}\left(\rho Y_{i} \mathbf{u}+\mathbf{J}_{i}\right) \cdot \mathbf{f}_{i}-\sum_{i=1}^{N} h_{i} w_{i}
$$

with $h_{i}=e_{i}+\frac{p_{i}}{\rho_{i}}$, where $e_{i}=e_{i}\left(\rho_{i}, \theta\right)$ and $p_{i}=p_{i}\left(\rho_{i}, \theta\right)$ define the thermodynamical state of the species $i$ (with $\rho_{i}=\rho Y_{i}$ ) The stress tensor is assumed to be of the form

$$
\sigma=-p\left(\rho, \theta,\left\{Y_{i}\right\}\right) \mathbf{1}+\mu \Delta \mathbf{u}+\nu \nabla(\operatorname{div} \mathbf{u}),
$$

where the total pressure is given by the Dalton's law

$$
p\left(\rho, \theta,\left\{Y_{i}\right\}\right)=\sum_{i=1}^{N} p_{i}\left(\rho, \theta, Y_{i}\right)
$$

and the heat flux is

$$
\mathbf{q}=-\lambda \nabla \theta+\sum_{i=1}^{N} h_{i} \mathbf{J}_{i}-p \sum_{i=1}^{N} D_{\theta, i} \mathbf{d}_{i}
$$


We consider the following diffusion model, generalizing the Fick's law, to fix the diffusion velocities

$$
\mathbf{J}_{i}=\sum_{j=1}^{N} C_{i j} \mathbf{d}_{j}-\rho Y_{i} D_{\theta, i} \frac{\nabla \theta}{\theta},
$$

where $C_{i j}$ are the multicomponent flux diffusion coefficients, $D_{\theta, i}$ is the thermal diffusion coefficient and $\mathbf{d}_{j}$ is the diffusion driving force $\mathbf{d}_{j}$ given by

$$
\mathbf{d}_{j}=\nabla X_{j}+\left(X_{j}-Y_{j}\right)\left(\frac{\nabla p}{p}\right)+\frac{\rho}{p} \sum_{k=1}^{N} Y_{j} Y_{k}\left(\mathbf{f}_{j}-\mathbf{f}_{k}\right),
$$

where the mole fraction of the species $i$ is

$$
X_{i}=\frac{Y_{i} / W_{i}}{\sum_{k=1}^{N} Y_{k} / W_{k}} .
$$

Finally let us suppose that the chemistry is described by a set of $M$ reactions

$$
\sum_{k=1}^{N} \nu_{i, k}^{\prime} \mathcal{M}_{i} \rightarrow \sum_{k=1}^{N} \nu_{i, k}^{\prime \prime} \mathcal{M}_{i} \text { for } k=1 \ldots M,
$$

where $\nu_{i, k}^{\prime}$ and $\nu_{i, k}^{\prime \prime}$ are the stoichiometric coefficients for species $i$, with chemical symbol $\mathcal{M}_{i}$ appearing as a reactant and as a product, respectively.

The corresponding reaction rates $w_{i}$ are given by different phenomenological expressions, depending on the physics involved.

In the combustion context chemical kinetics [51] using Arrhenius and mass action laws one takes generally

$$
w_{i}=W_{i} \sum_{k=1}^{M}\left(\nu_{i, k}^{\prime \prime}-\nu_{i, k}^{\prime}\right) B_{k} \theta^{\alpha_{k}} e^{-\frac{E_{k}}{R^{0} \theta}} \prod_{j=1}^{N}\left(\frac{\rho Y_{j}}{W_{j}}\right)^{\nu_{j, k}^{\prime}} \text { for } k=1 \ldots N .
$$

In the astrophysical context [30]

$$
w_{i}=-\mathcal{R}_{i} Y_{i}+\sum_{j=1}^{N} \mathcal{R}_{i j} Y_{i} Y_{j}+\sum_{k, l=1}^{N} \mathcal{R}_{k l} Y_{k} Y_{l}+\cdots,
$$

where $\mathcal{R}_{i}>0$ characterizes the photodisintegrations ( $\beta$-decays) of nucleus $i, \mathcal{R}_{a b}$ is the rate, per unit of time and volume, of destruction (if $a=i$ or $b=i$ ) or production (if $a \neq i$ and $b \neq i$ ) of species $i$ by a (two-body) nuclear reaction, and $\cdots$ is the contribution of $N$-body reactions with $N>2$. All these coefficients are generally strongly dependent on $\rho, \theta$.

Although a lot of its properties have been studied by Giovangigli [34], the previous system seems to be presently out of reach of mathematical treatment, at least concerning the reacting case. However, for a multicomponent model involving only thermodiffusion coupling, it is possible to prove a local existence result, extending the method of Tabata $[49]$. 
3. Simplified models for a binary mixture. We first consider, as a simple model, the first equation of the PP II cycle

$$
{ }^{3} \mathrm{He}+{ }^{4} \mathrm{He} \rightarrow{ }^{7} \mathrm{Be}+\gamma .
$$

We also suppose that the reaction takes place in the presence of neutrons acting as a dilutant, so this unique reaction $(M=1)$ implies $N=4$ species (discarding the massless photons), and we suppose that the field force does not depend on the species $\mathbf{f}_{i} \equiv \mathbf{f}=$ $-G \nabla \Phi$ (gravitation). The system reads

$$
\left\{\begin{array}{l}
\partial_{t} \rho+\nabla \cdot(\rho \mathbf{u})=0, \\
\partial_{t}(\rho \mathbf{u})+\nabla \cdot(\rho \mathbf{u} \otimes \mathbf{u})=\nabla \cdot \sigma+\rho \mathbf{f}, \\
\partial_{t}(\rho e)+\nabla \cdot(\rho e \mathbf{u})=\sigma: \nabla \mathbf{u}-\nabla \cdot \mathbf{q}+\rho \mathbf{f} \cdot \mathbf{u}+\mathbf{f} \cdot \sum_{i=1}^{N} \mathbf{J}_{i}-\sum_{j=1}^{4} h_{i}^{0} w_{i}, \\
\partial_{t}\left(\rho Y_{i}\right)+\nabla \cdot\left(\rho Y_{i} \mathbf{u}\right)=w_{i}-\nabla \cdot \mathbf{J}_{i},
\end{array}\right.
$$

for $i={ }^{3} \mathrm{He},{ }^{4} \mathrm{He},{ }^{7} \mathrm{Be}, n$ (with $Y^{3} \mathrm{He}+Y_{4}{ }_{\mathrm{He}}+Y_{{ }^{7} \mathrm{Be}}+Y_{n}=1$ ), where the fluxes are

$$
\mathbf{J}_{i}=\sum_{j=1}^{4} C_{i j} \mathbf{d}_{j}-\rho Y_{i} D_{\vartheta, i} \frac{\nabla \theta}{\theta},
$$

where the last term corresponds to a diffusion arising from a temperature gradient (Soret effect), and

$$
\begin{gathered}
\mathbf{d}_{j}=\nabla X_{j}+\left(X_{j}-Y_{j}\right) \frac{\nabla p}{p}, \\
\mathbf{q}=-\lambda \nabla \vartheta+\sum_{i=1}^{4} h_{i} \mathbf{J}_{i}-p \sum_{i=1}^{4} D_{\theta, i} \mathbf{d}_{i},
\end{gathered}
$$

where the last term corresponds to heat diffusion arising from a concentration gradient (Dufour effect).

The chemical source terms are

$$
\left\{\begin{array}{l}
w_{{ }^{3} H e}=-W_{{ }^{3} H e} \Phi, \\
w_{{ }_{H} H_{e}}=-W_{{ }^{4} H e} \Phi, \\
w_{\mathrm{7} B e}=W_{{ }^{B} \mathrm{Be}} \Phi,
\end{array}\right.
$$

where

$$
\Phi:=B \vartheta^{\alpha} e^{-\frac{E}{R^{0} \vartheta}} \frac{\rho Y^{3} H e}{W_{3} H e} \frac{\rho Y_{4} H e}{W_{4} H e} .
$$

If we neglect the Soret and Dufour effects and if we suppose $C_{i j}=C \delta_{i j}$, and that the species specific heats at constant pressure are constant and independent of temperature, we get for $\mathbf{J}_{i}$ the pure Fick's law

$$
\mathbf{J}_{i}=-\rho D_{i} \nabla Y_{i}
$$

where $D_{i}$ is the empirical diffusion coefficient of species $i$ and

$$
\mathbf{q}=-\lambda \nabla \vartheta
$$


Then we get the system

$$
\left\{\begin{array}{l}
\partial_{t} \rho+\nabla \cdot(\rho \mathbf{u})=0 \\
\partial_{t}(\rho \mathbf{u})+\nabla \cdot(\rho \mathbf{u} \otimes \mathbf{u})=\nabla \cdot \sigma+\rho \mathbf{f} \\
\partial_{t}(\rho e)+\nabla \cdot(\rho e \mathbf{u})=\sigma: \nabla \mathbf{u}+\nabla \cdot(\lambda \nabla \vartheta)+\rho \mathbf{f} \cdot \mathbf{u}-\sum_{j=1}^{4} h_{i}^{0} w_{i} \\
\partial_{t}\left(\rho Y_{i}\right)+\nabla \cdot\left(\rho Y_{i} \mathbf{u}\right)=w_{i}+\nabla \cdot\left(D_{i} \nabla Y_{i}\right)
\end{array}\right.
$$

for $i={ }^{3} \mathrm{He},{ }^{4} \mathrm{He},{ }^{7} \mathrm{Be}, n$.

If we further assume that ${ }^{3} \mathrm{He}$ and ${ }^{7} \mathrm{Be}$ are present only by trace amounts in the mixture, and that the ${ }^{4} \mathrm{He}$ and the neutrons are in excess, we can suppose that $Y_{4}{ }_{\mathrm{He}}$ and $Y_{n}$ are constant and then we write

$$
\left\{\begin{array}{l}
Y_{{ }^{4} \mathrm{He}}=Y_{{ }^{H} \mathrm{He}}^{0} \\
Y_{n}=Y_{n}^{0} \\
Y_{{ }^{H} \mathrm{He}}<<Y_{{ }^{0} \mathrm{He}}^{0}+Y_{n}^{0} \\
Y_{{ }^{B} \mathrm{Be}}<<Y_{{ }_{H e}}^{0}+Y_{n}^{0}
\end{array}\right.
$$

and the equations for $Y_{4}{ }_{\mathrm{He}}$ and $Y_{n}$ are decoupled. The only remaining equation is that corresponding to ${ }^{3} \mathrm{He}$ (with $Z:=Y^{3} \mathrm{He}$ ) and we recover the simplest combustion model for reacting fluids [4]

$$
\begin{gathered}
\partial_{t} \varrho+\nabla \cdot(\varrho \mathbf{u})=0 \\
\partial_{t}(\varrho \mathbf{u})+\operatorname{div}(\varrho \mathbf{u} \otimes \mathbf{u})+\nabla_{x} p=\operatorname{div} \mathbb{S}+\varrho \nabla_{x} \Psi \\
\partial_{t}(\varrho e)+\operatorname{div}(\varrho e \mathbf{u})+\operatorname{div} \mathbf{q}=\Phi-p \operatorname{div} \mathbf{u}+h w(\varrho, \vartheta, Z), \\
-\Delta \Psi=G \varrho \text { on } R^{3} \\
\partial_{t}(\varrho Z)+\operatorname{div}(\varrho Z \mathbf{u})=-\operatorname{div} \mathbf{F}+w(\varrho, \vartheta, Z)
\end{gathered}
$$

where the chemical source $w$ is

$$
w(Z, T):=-\varrho Z A
$$

where $A$ is the (positive) reaction rate.

From the previous considerations, we have to solve the system (10)-(14) for the density $\varrho=\varrho(t, x)$, the velocity $\mathbf{u}=\mathbf{u}(t, x)$, the absolute temperature $\vartheta=\vartheta(t, x)$, and the concentration $Z$, evaluated at the time $t \in[0, T]$ and the reference spatial position $x \in$ $\Omega \subset R^{3}$.

In (13) $p$ is the pressure, $\mathbb{S}$ stands for the viscous stress tensor, and $\nabla_{x} \Psi$ is the extraneous force acting on the mixture due to self-gravitation, where the potential $\Psi$ solves the Poisson equation (13) where $\varrho$ is extended to be zero outside $\Omega$.

In (12) $\mathbf{q}$ denotes the heat energy flux, $\Phi$ is the dissipation function representing the irreversible transfer of the mechanical energy into heat and $h$ is a positive constant.

In (14) $\mathbf{F}$ is the diffusion flux. 
If the motion is smooth enough, the momentum equation (11) can be multiplied on $\mathbf{u}$ in order to obtain

$$
\begin{gathered}
\partial_{t}\left(\frac{1}{2} \varrho|\mathbf{u}|^{2}-\frac{G}{2} \Delta^{-1}[\varrho] \varrho\right)+\operatorname{div}\left(\left(\frac{1}{2} \varrho|\mathbf{u}|^{2}+p\right) \mathbf{u}\right) \\
=\operatorname{div}(\mathbb{S u})+p \operatorname{div} \mathbf{u}-\mathbb{S}: \nabla_{x} \mathbf{u}+\operatorname{div}(\varrho \Psi \mathbf{u})
\end{gathered}
$$

where $\frac{1}{2} \varrho|\mathbf{u}|^{2}$ denotes the kinetic energy.

The total energy $E \equiv \frac{1}{2} \varrho|\mathbf{u}|^{2}+\varrho e+h \varrho Z-\frac{1}{2} \varrho G \Delta^{-1}[\varrho] \varrho+h \varrho Z$ being a conserved quantity

$$
\frac{d}{d t} \int_{\Omega} E(t) d x=0
$$

we get

$$
\Phi=\mathbb{S}: \nabla_{x} \mathbf{u}
$$

provided the motion characterized by the velocity $\mathbf{u}$ is smooth. In general, one can assert only

$$
\Phi \geq \mathbb{S}: \nabla_{x} \mathbf{u}
$$

Finally, assuming that $p=p(\varrho, \vartheta)$ and $e=e(\varrho, \vartheta)$ are explicit functions of the density, the absolute temperature, we get the specific entropy through the thermodynamical relationship:

$$
\frac{\partial s}{\partial \varrho}=\frac{1}{\vartheta}\left(\frac{\partial e}{\partial \varrho}-\frac{p}{\varrho^{2}}\right), \frac{\partial s}{\partial \vartheta}=\frac{1}{\vartheta} \frac{\partial e}{\partial \vartheta},
$$

Accordingly, the internal energy balance (12) may be put into an equivalent form of the entropy equation

$$
\partial_{t}(\varrho s)+\operatorname{div}(\varrho s \mathbf{u})+\operatorname{div}\left(\frac{\mathbf{q}}{\vartheta}\right)=\frac{\Phi}{\vartheta}-\frac{\mathbf{q} \cdot \nabla_{x} \vartheta}{\vartheta^{2}}+h \frac{w}{\vartheta} .
$$

By virtue of the Clausius-Duhem inequality, the right-hand side of (20) must be non-negative for any possible motion, so

$$
\Phi \geq \mathbb{S}: \nabla_{x} \mathbf{u} \geq 0, \mathbf{q} \cdot \nabla_{x} \vartheta \leq 0 .
$$

We suppose tha the state variables $\varrho$ and $\vartheta$, including the radiation (taking into account the photons $\gamma$ in (7)), is provided by the general form:

$$
p=p_{G}+p_{R},
$$

with a gaseous contribution

$$
p_{G}=p_{e}(\varrho)+\vartheta p_{\vartheta}(\varrho),
$$

where the elastic pressure $p_{e}$ as well as the thermal pressure component $p_{\vartheta}$ are continuously differentiable functions of the density, and we assume that

$$
\begin{gathered}
p_{e}(0)=0, p_{e}^{\prime}(\varrho) \geq a_{1} \varrho^{\gamma-1}-c_{1}, p_{e}(\varrho) \leq a_{2} \varrho^{\gamma}+c_{2}, \\
p_{\vartheta}(0)=0, p_{\vartheta}^{\prime}(\varrho) \geq 0, p_{\vartheta}(\varrho) \leq a_{3} \varrho^{\Gamma}+c_{3},
\end{gathered}
$$

with

$$
a_{1}>0, \gamma \geq 2, \gamma>\frac{4 \Gamma}{3}
$$


The radiative pressure term has the form

$$
p_{R}=\frac{d}{3} \vartheta^{4}
$$

where $d>0$ is the Stefan-Boltzmann constant. Consequently, we have

$$
p=p(\varrho, \vartheta)=p_{G}(\varrho, \vartheta)+p_{R}(\varrho, \vartheta)=p_{e}(\varrho)+\vartheta p_{\vartheta}(\varrho)+\frac{d}{3} \vartheta^{4} .
$$

According (19), the corresponding form of the internal energy density reads

$$
e(\varrho, \vartheta)=P_{e}(\varrho)+d \frac{\vartheta^{4}}{\varrho}+Q(\vartheta)
$$

where $Q$ is a non-decreasing function of the temperature. More specifically, the quantity

$$
c_{v}(\vartheta)=Q^{\prime}(\vartheta)
$$

is termed the specific heat at constant volume.

For the sake of simplicity, we shall assume $c_{v}$ to be a positive constant, which yields

$$
s(\varrho, \vartheta)=\frac{4}{3} d \frac{\vartheta^{3}}{\varrho}-P_{\vartheta}(\varrho)+c_{v} \log (\vartheta),
$$

where

$$
P_{e}(\varrho)=\int_{1}^{\varrho} \frac{p_{e}(z)}{z^{2}} d z, P_{\vartheta}(\varrho)=\int_{1}^{\varrho} \frac{p_{\vartheta}(z)}{z^{2}} d z .
$$

We restrict now the study to Newtonian fluids for which the viscosity tensor $\mathbb{S}$ is a linear function of the velocity gradient,

$$
\mathbb{S}=\mu\left(\nabla_{x} \mathbf{u}+{ }^{t} \nabla_{x} \mathbf{u}\right)+\lambda \operatorname{div} \mathbf{u} \mathbb{I},
$$

with the shear viscosity coefficient $\mu$, and the bulk viscosity coefficient $\zeta=\lambda+\frac{2}{3} \mu$. After (21), both $\mu$ and $\zeta$ must be non-negative.

The heat flux $\mathbf{q}$ will be taken through the classical Fourier law:

$$
\mathbf{q}=-\kappa \nabla_{x} \vartheta
$$

with the heat conductivity coefficient $\kappa$.

For the diffusion flux $\mathbf{F}$, we follow the Fick's law

$$
\mathbf{F}=-\varrho d \kappa \nabla_{x} Z a
$$

with the diffusion coefficient $d$.

As generally, both viscosity and heat conductivity of a gas depend on the temperature, we suppose that

$$
\left\{\begin{array}{c}
\left.0<\underline{\mu}\left(1+\vartheta^{\beta}\right) \leq \mu(\vartheta), \quad\left|\mu^{\prime}(\vartheta)\right| \leq \bar{\mu}(1+\vartheta)^{\beta-1}\right), \\
\left.\zeta \geq 0, \quad \underline{\zeta} \vartheta^{\beta}-1 \leq \zeta(\vartheta), \quad\left|\zeta^{\prime}(\vartheta)\right| \leq \bar{\zeta}(1+\vartheta)^{\beta-1}\right) .
\end{array}\right\}
$$

Similarly, we set

$$
\kappa=\kappa_{G}(\vartheta)+\kappa_{R}(\varrho, \vartheta)
$$

with

$$
0<\underline{\kappa}_{G} \leq \kappa_{G}(\vartheta) \leq \bar{\kappa}_{G}\left(1+\vartheta^{\alpha}\right)
$$


and the radiative term

$$
\underline{\kappa} \vartheta^{\alpha} \leq \kappa_{R}(\varrho, \vartheta) \leq \bar{\kappa}\left(1+\vartheta^{\alpha}\right) .
$$

As a matter of fact, the quantity $\sigma$ itself depends on $\varrho$ and $\vartheta$ in a rather complicated way. For the sake of simplicity, however, we consider the "zero order" approximation taking $\sigma$ a positive constant).

Finally, the flux diffusion coefficient $D=\varrho d$ is supposed to be a continuously differentiable function depending on the temperature, such that

$$
0 \leq \underline{D}(\vartheta) \leq \bar{D}\left(1+\vartheta^{\beta}\right), \text { for all } \vartheta>0,
$$

with positive coefficients $\underline{D}$ and $\bar{D}$, and the reaction rate is also supposed to be a continuously differentiable function depending on the temperature, such that

$$
0 \leq A(\vartheta) \leq \bar{A} \vartheta, \text { for all } \vartheta>0,
$$

with positive coefficient $\bar{A}$.

In order to obtain, at least formally, a well-posed problem, we take a no-slip boundary condition for the velocity field:

$$
\left.\mathbf{u}\right|_{\partial \Omega}=0,
$$

a thermally isolate condition for the temperature

$$
\left.\mathbf{q} \cdot \mathbf{n}\right|_{\partial \Omega}=0,
$$

where $\mathbf{n}$ denotes the outer normal vector, and a no-diffusion condition for the diffusion flux

$$
\left.\mathbf{F} \cdot \mathbf{n}\right|_{\partial \Omega}=0
$$

However, it is not known if, given the initial state variables:

$$
\varrho(0, \cdot)=\varrho_{0}, \varrho \mathbf{u}(0, \cdot)=\mathbf{m}_{0}, \vartheta(0, \cdot)=\vartheta_{0}, Z(0, \cdot)=Z_{0},
$$

a classical (smooth) solution of the problem satisfying the initial conditions (43) exists on an arbitrarily large time interval $(0, T)$.

In order to avoid this difficulty, it is shown in [28] how to develop a mathematical theory based on the concept of variational solutions(recall that the first rigorous results for compressible fluids were presented by P.-L. Lions [43] and see also [23] for the astrophysical non-reactive context).

In the present reactive case, we say that the functions

$$
\varrho \in L^{\infty}\left(0, T ; L^{\gamma}(\Omega)\right), \quad \mathbf{u} \in L^{2}\left(0, T ; W_{0}^{1,2}\left(\Omega ; \mathbb{R}^{3}\right)\right), \quad \vartheta \in L^{2}\left(0, T ; W^{1,2}(\Omega)\right),
$$

and

$$
Z \in L^{\infty}((0, T) ; \Omega) \cap L^{2}\left(0, T ; W^{1,2}(\Omega)\right),
$$

represent a variational solution of the problem (10)-(14), (16), (43), if

1. the density $\varrho$ is non-negative on $(0, T) \times \Omega$,

$$
\log \vartheta \in L^{2}\left(0, T ; W^{1,2}(\Omega)\right),
$$

and

$$
0 \leq Z(t, x) \leq 1 \quad \text { for a.a. }(t, x) \in(0, T) \times \Omega
$$


2. the continuity equation (10) is satisfied in the distribution sense $\mathcal{D}^{\prime}\left(0, T \times \mathbb{R}^{3}\right)$, provided that $\varrho, \mathbf{u}$ are zero outside $\Omega$.

3. the dynamical equation $(11)$ holds in $\mathcal{D}^{\prime}((0, T) \times \Omega)$ and the gravitational potential $\Phi$ is obtained from the Poisson equation (13) considered on $\mathbb{R}^{3}$,

4. the specific entropy $s$ satisfies the inequality

$$
\begin{aligned}
& \int_{0}^{T} \int_{\Omega} \varrho s \partial_{t} \varphi+\varrho s \mathbf{u} \cdot \nabla_{x} \varphi+\frac{\mathbf{q}}{\vartheta} \cdot \nabla_{x} \varphi d x d t \leq \\
& \int_{0}^{T} \int_{\Omega}\left(\frac{\mathbf{q} \cdot \nabla_{x} \vartheta}{\vartheta^{2}}-\frac{\mathbb{S}: \nabla_{x} \mathbf{u}}{\vartheta}\right) \varphi+\frac{h \varrho Z A}{\vartheta} d x d t
\end{aligned}
$$

for any non-negative function $\varphi \in \mathcal{D}((0, T) \times \bar{\Omega})$,

5 . the reactant fraction $Z$ satisfies the identity

$$
\begin{gathered}
\int_{0}^{T} \int_{\Omega} \varrho Z \partial_{t} \varphi+\varrho Z \mathbf{u} \cdot \nabla_{x} \varphi-\varrho d \nabla_{x} Z \cdot \nabla_{x} \varphi d x d t= \\
\int_{0}^{T} \int_{\Omega} \varrho Z A \varphi d x d t
\end{gathered}
$$

for any function $\varphi \in \mathcal{D}((0, T) \times \bar{\Omega})$,

6. the total energy $E$ is a constant of motion:

$$
\int_{\Omega} E\left(t_{1}\right) d x=\int_{\Omega} E\left(t_{2}\right) d x, \quad \text { for any Lebesgue points } t_{1}, t_{2} \in(0, T) .
$$

One can check that for any variational solution of the previous problem, the internal energy equation

$$
\partial_{t}(\varrho e)+\operatorname{div}(\varrho e \mathbf{u})+\operatorname{divq}=\mathbb{S}: \nabla_{x} \mathbf{u}-p \operatorname{div} \mathbf{u}+h \varrho Z A
$$

holds in $(0, T) \times \bar{\Omega})$.

Under the previous conditions, the following result due to E. Feireisl and A. Novotny [29] holds (see also D. Donatelli and K. Trivisa [16] for different hypotheses),

TheOREM 1. Let $\Omega \subset R^{3}$ be a bounded domain with a boundary of class $C^{2+\nu}, \nu>0$. Suppose that the pressure $p$ is determined through the state equation (27), with $d>0$, and $p_{e}, p_{\vartheta}$ satisfying (23 - 25). Furthermore, let the viscous stress tensor $\mathbb{S}$ be given by (31), where $\mu$ and $\zeta$ are continuous differentiable globally Lipschitz functions of $\vartheta$ obeying (34) for $0 \leq \alpha \leq \frac{4}{3}$.

Let $\mathbf{q}$ be given by (32) with $\kappa$ satisfying (35 - 37) for $\alpha \geq \frac{16}{3}-\beta$, and similarly, let $D=\varrho d$ be given by (38) and the reaction rate $A$ be given by (39).

Finally, assume the initial data $\varrho_{0}, \mathbf{u}_{0}, \vartheta_{0}, Z_{0}$ satisfy

$$
\left\{\begin{array}{c}
\left.\varrho_{0}, \vartheta_{0}, Z_{0} \in L^{\infty}(\Omega), \mathbf{u}_{0} \in L^{\infty}(\Omega) ; \mathbb{R}^{3}\right), \\
\vartheta_{0} \geq 0, \quad 0<\underline{\vartheta} \leq \vartheta(x) \leq \bar{\vartheta}, \\
0 \leq Z(x) \leq 1, \text { for a.a. } x \in \Omega .
\end{array}\right\}
$$

Then for any given $T>0$, the problem (10)-(14), (16), (43), (40), (41), (42) possesses a variational solution on the set $(0, T) \times \Omega$ in the sense of the previous definition, moreover 
this solution satisfies the initial conditions

$$
\left\{\begin{array}{c}
\varrho(t) \rightarrow \varrho_{0}, i n L^{1}(\Omega), \\
(\varrho Z)(t) \rightarrow \varrho_{0} Z_{0} \text { weakly in } L^{1}(\Omega),
\end{array}\right\}
$$

as $t \rightarrow 0+$, moreover

$$
\text { ess } \lim _{t \rightarrow 0+} \int_{\Omega}(\varrho s)(t) \phi d x \geq \int_{\Omega} \varrho_{0} s_{0} \phi d x,
$$

for any $\phi \geq 0, \phi \in \mathcal{D}^{\prime}(\Omega)$, where

$$
\varrho_{0} s_{0}=\frac{4 d}{3} \vartheta_{0}^{3}-\varrho_{0} P_{\vartheta}\left(\varrho_{0}\right)+c_{v} \varrho_{0} \log \left(\vartheta_{0}\right) .
$$

4. A one-dimensional model. Our purpose in this section is to consider a simplified model of multicomponent fluid with a unique chemical reaction, in the framework of 1D-flows.

We suppose that the species $E_{1}, \ldots, E_{N}$ interact through the chemical reaction

$$
\sum_{i=1}^{N} \alpha_{i} E_{i} \rightarrow \sum_{i=1}^{N} \beta_{i} E_{i}
$$

where $\alpha_{i}, \beta_{i}$ are the stoichiometric coefficients of species $i$ in the chemical reaction.

The problem under study is then the following compressible Navier-Stokes system in the lagrangian setting [1] describing 1D-flows of a viscous heat-conducting radiative and reactive gas for a $N$ species mixture

$$
\begin{gathered}
\eta_{t}=v_{x}, \\
v_{t}=\sigma_{x}, \\
e_{t}=q_{x}+\sigma v_{x}, \\
\left(Y_{i}\right)_{t}=\left(J_{i}\right)_{x}+\omega_{i}, \quad i=1, \ldots, N,
\end{gathered}
$$

for $(x, t) \in Q \equiv \Omega \times \mathbf{R}^{+}=(0, M) \times(0, \infty)$, where $x, t$ are the Lagrangian mass coordinates.

The first equations (50)-(52) describe the flow of the mixture (specific volume $\eta$, velocity $v$, temperature $\theta$ ) and the set of equations (53) for $i=1, \ldots, N$ is a reactiondiffusion system describing the concentrations $Y_{i}$ of the individual species.

We choose the state functions $p$ (pressure), $e$ (specific internal energy) and $s$ (specific entropy) of the form

$$
p\left(\eta, \theta,\left\{Y_{i}\right\}_{i=1, \ldots, N}\right)=\sum_{i=1}^{N} \frac{R_{i} Y_{i} \theta}{\eta}+\frac{a}{3} \theta^{4},
$$

and

$$
\begin{gathered}
e\left(\eta, \theta,\left\{Y_{i}\right\}_{i=1, \ldots, N}\right)=\sum_{i=1}^{N} c_{V i} Y_{i} \theta+a \eta \theta^{4} . \\
s\left(\eta, \theta,\left\{Y_{i}\right\}_{i=1, \ldots, N}\right)=\sum_{i=1}^{N}\left(R_{i} \log \eta_{i}+c_{V i} \log \theta\right)+\frac{4}{3} a \eta \theta^{3},
\end{gathered}
$$

with $\eta_{i}=\frac{1}{\rho_{i}}$ and $\rho_{i}=\rho Y_{i}$. 
This choice corresponds to a mixture of perfect gases with a radiating part (photon gas) in local thermodynamical equilibrium.

For the stress we take a linearly viscous model for the mixture

$$
\sigma\left(\eta, \theta,\left\{Y_{i}\right\}_{i=1, \ldots, N}\right)=-p\left(\eta, \theta,\left\{Y_{i}\right\}_{i=1, \ldots, N}\right)+\nu \rho v_{x}
$$

where $\rho=\frac{1}{\eta}$.

The heat flux is

$$
q=-\kappa \rho \theta_{x}+\sum_{i=1}^{N} \rho Y_{i} V_{i} h_{i}
$$

where the diffusion velocities follow the simple Fick's law

$$
V_{i}=-D_{i} \frac{\left(Y_{i}\right)_{x}}{Y_{i}}
$$

with the individual enthalpy $h_{i}=c_{P i} \theta$.

We adopt a simple form for the diffusion flux (see LANDAU [40] for more general models and [49] for a mathematical analysis of these models)

$$
J_{i}=\rho Y_{i} V_{i}
$$

The molar production rates $\omega_{i}$ for $i=1, \ldots, N$ are given functions of the form

$$
\begin{gathered}
\omega_{i}\left(\left\{\alpha_{j}, \beta_{j}\right\}_{j=1, \ldots, N} ; \eta, \theta,\left\{Y_{j}\right\}_{j=1, \ldots, N}\right) \\
=A_{i} \theta^{a_{i}} e^{-\frac{e_{i}}{r \theta}} \rho^{b} \tilde{\omega}_{i}\left(\left\{\alpha_{j}, \beta_{j}\right\}_{j=1, \ldots, N} ;\left\{Y_{j}\right\}_{j=1, \ldots, N}\right)
\end{gathered}
$$

where the coefficients $A_{i}, a_{i}, e_{i}, r, b$ are positive and the functions $\tilde{\omega}_{i}$ depending on the $Y_{i}$ and the stoichiometric coefficients $\alpha_{i}, \beta_{i}$, may be negative (destruction of species $i$ ) or positive (production of species $i$ ).

More precisely we assume that

$$
\tilde{\omega}_{i}\left(\left\{\alpha_{j}, \beta_{j}\right\}_{j=1, \ldots, N-1} ;\left\{Y_{j}\right\}_{j=1, \ldots, N-1}\right) \leq 0, \text { for } 1 \leq i \leq N-1 .
$$

Moreover we suppose that the $\omega_{i}$ satisfy the global mass conservation

$$
\sum_{i=}^{N} \omega_{i}=0
$$

Finally, it will be useful to consider the evolution equation for the balance of entropy

$$
s_{t}+\left(j_{S}\right)_{x}=\varsigma_{S},
$$

where the entropy flux $j_{S}$ is

$$
j_{S}=\frac{1}{\theta}\left(q-\sum_{i=1}^{N} \rho Y_{i} V_{i} \mu_{i}\right)
$$

the entropy source is

$$
\varsigma_{S}=-\frac{1}{\theta^{2}} q \theta_{x}-\sum_{i=1}^{N} \rho Y_{i} V_{i}\left(\frac{\mu_{i}}{\theta}\right)_{x}+\frac{1}{\theta} \nu \rho v_{x}^{2}-\frac{1}{\theta} \sum_{i=1}^{N} \mu_{i} \omega_{i},
$$


where the chemical potential $\mu_{i}$ is computed using the thermodynamical Gibbs relation

$$
\theta d s=d e+p d\left(\frac{1}{\rho}\right)-\sum_{i=1}^{N} \mu_{i} Y_{i} .
$$

For the perfect gas $i$, the chemical potential $\mu_{i}$ is [5]

$$
\mu_{i}\left(\eta, \theta, Y_{i}\right)=\mu_{i}^{0}+\log \frac{p_{i}}{p_{0}}
$$

with $p_{i}(\eta, \theta)=\frac{R_{i} Y_{i} \theta}{\eta}$, for an arbitrary reference state $\left(\mu_{i}^{0}, p_{0}\right)$.

We take the following boundary conditions

$$
\begin{gathered}
\left.v\right|_{x=0}=0,\left.\quad \sigma\right|_{x=M}=-p_{\Gamma}, \\
\left.q\right|_{x=0}=0,\left.\quad \theta\right|_{x=M}=\theta_{\Gamma}, \\
\left.J_{i}\right|_{x=0, M}=0, \text { for } i=1, \ldots, N-1 .
\end{gathered}
$$

with given $p_{\Gamma}=$ const $>0$ and $\theta_{\Gamma}=$ const $>0$, together with the initial conditions

$$
\left.\left(\eta, v, \theta, Y_{i}\right)\right|_{t=0}=\left(\eta^{0}(x), v^{0}(x), \theta^{0}(x), Y_{i}^{0}(x)\right), \text { for } x \in \Omega, \quad i=1, \ldots, N .
$$

The unknown quantities $\eta>0, v, \theta>0$ and $0 \leq Y_{i} \leq 1$ are the specific volume, the velocity, the absolute temperature and the concentration of species $i$, with the mass constraint

$$
\sum_{i=1}^{N} Y_{i}=1
$$

Finally $\kappa(\eta, \theta), p_{\Gamma}$ and $\theta_{\Gamma}$ are the heat conductivity, the outer pressure and the outer temperature, and the quantities $\nu, R_{i}, a, c_{V i}, c_{P i}, D_{i}, \nu$ and $d$ are positive physical constants as well as $M$ is the total mass of the gas.

In the simple case of section 3, for arbitrarily large data, it is possible to prove [26] (see also the references therein and [3] [4] [8] [12] [13] [14] [19] [20] [35] [52] for related works on this model) global (in time) bounds for the solution of the above problem and establish its global exponential decay as $t \rightarrow \infty$ in suitable spaces.

We show in the following that the Lyapunov functional method may be partially extended to the multicomponent case.

4.1. Statement of the results. In the following, we use the classical Lebesgue and Sobolev spaces $L^{q}(G)$ and $W^{1, q}(G)$, for $q \in[1, \infty]$, with $H^{1}(G)=W^{1,2}(G)$ and $H^{0}(G)=L^{2}(G)$. We also need the anisotropic Lebesgue and Sobolev spaces $L^{q, r}\left(Q_{T}\right)$ and $H^{2,1}\left(Q_{T}\right) \equiv$ $W_{2}^{2,1}\left(Q_{T}\right)$, for $Q_{T}:=\Omega \times(0, T)$ with $0<T \leq \infty$ (see [41]), with the norms

$$
\begin{gathered}
\|w\|_{L^{q, r}\left(Q_{T}\right)}=\|\| w\left\|_{L^{q}(\Omega)}\right\|_{L^{r}(0, T)}, \\
\|w\|_{H^{2,1}\left(Q_{T}\right)}=\|w\|_{V_{2}\left(Q_{T}\right)}+\left\|w_{x}\right\|_{V_{2}\left(Q_{T}\right)}+\left\|w_{t}\right\|_{L^{2}\left(Q_{T}\right)},
\end{gathered}
$$

where $V_{2}\left(Q_{T}\right)$ is the standard space of functions $w$ on $Q_{T}$ having finite (parabolic) energy $\|w\|_{V_{2}\left(Q_{T}\right)}=\|w\|_{L^{2, \infty}\left(Q_{T}\right)}+\left\|w_{x}\right\|_{L^{2}\left(Q_{T}\right)}$. We define the following integration operators, for $(x, t) \in \bar{Q}$ 


$$
(I \varphi)(x)=\int_{0}^{x} \varphi(\xi) d \xi,\left(I^{*} \varphi\right)(x)=\int_{x}^{M} \varphi(\xi) d \xi,\left(I_{0} y\right)(t)=\int_{0}^{t} y(\tau) d \tau
$$

and denote by $\langle\varphi\rangle=\frac{1}{M} \int_{\Omega} \varphi(x) d x$ the mean value over $\Omega$, for any $\varphi \in L^{1}(\Omega)$.

We assume that $\kappa(\eta, \theta)$ is strictly positive and continuously differentiable on $\mathbf{R}^{+} \times \overline{\mathbf{R}}^{+}$, moreover we suppose that

$$
\kappa_{1} \eta+\kappa_{2} \leq \kappa(\eta, \theta)
$$

where $\kappa_{1}>0$ and $\kappa_{2}>0$.

We limit ourselves to the case of global solution with strong components $(\eta, v, \theta)$ such that

$$
\eta, \eta_{x}, \eta_{t} \in L^{2, \infty}\left(Q_{T}\right), \min _{\bar{Q}_{T}} \eta>0 ; v, \theta \in H^{2,1}\left(Q_{T}\right), \theta>0 \text { in } \bar{\Omega} \times(0, T],
$$

and $Y_{i} \in V_{2}\left(Q_{T}\right)$, for any $i=1, \ldots, N$ and any $T<\infty$. The following requirements on the data are necessary

$$
\eta^{0}, v^{0}, \theta^{0} \in H^{1}(\Omega), \min _{\bar{\Omega}} \eta^{0}>0, \theta^{0} \geq 0, v^{0}(0)=0, \theta^{0}(M)=\theta_{\Gamma} .
$$

We also suppose that $Y_{i}^{0} \in L^{\infty}(\Omega)$, for any $i=1, \ldots, N$.

Let us start with the stationary problem, defined by the system

$$
\left\{\begin{array}{l}
v_{x}=0, \\
q_{x}=0, \\
\left(J_{i}\right)_{x}+\omega_{i}=0, \quad i=1, \ldots, N,
\end{array}\right.
$$

together with (57), for $x \in \Omega$, and also with the first relation (54), under the boundary conditions (68)-(70).

Proposition 1. Suppose that the chemical sources $\omega_{i}$ satisfy the additional condition

"The algebraic system

$$
\tilde{\omega}_{i}\left(\left\{\alpha_{j}, \beta_{j}\right\}_{j=1, \ldots, N-1} ;\left\{Y_{j}\right\}_{j=1, \ldots, N-1}\right)=0, \quad i=1, \ldots, N-1,
$$

has a unique solution $\left(y_{1}, \ldots y_{N-1}\right) \in \mathbf{R}^{N-1}$, with $0 \leq y_{i} \leq 1$, for $i=1, \ldots, N-1$."

Suppose also that the condition

$$
\frac{a}{3} \theta_{\Gamma}^{4}<p_{\Gamma}
$$

is valid.

Then, the weak solution $\eta_{S}, v_{S}, \theta_{S}, Y_{i S}, i=1, \ldots, N$ in $W^{1,1}(\Omega)$ of the stationary problem satisfying $\min _{\bar{\Omega}} \eta_{S}>0$ and $\theta_{S} \geq 0$ exists, and under conditions (76) and (77), the solution is such that

$$
\left\{\begin{array}{c}
Y_{i S}(x) \equiv y_{i}, \quad i=1, \ldots, N \\
v_{S}(x) \equiv 0 \\
\theta_{S}(x) \equiv \theta_{\Gamma} \\
\rho_{S}(x) \equiv \frac{p_{\Gamma}-\frac{a}{3} \theta_{\Gamma}^{4}}{\sum_{i=1}^{N} R_{i} y_{i} \theta_{\Gamma}}
\end{array}\right.
$$

with $\rho_{S}=\frac{1}{\eta_{S}}$ and $y_{N}=1-\sum_{i=1}^{N-1} y_{i}$. 
Proof. As $\omega_{i} \leq 0$ for $i=1, \ldots, N-1$, multiplying the last equation (75) by $Y_{i}$ and integrating by parts on $\Omega$, we find

$$
\int_{\Omega} \rho_{S}(x) D_{i} Y_{i S}^{2}(x) d x \leq 0,
$$

for $i=1, \ldots, N-1$, which implies that $Y_{i S}=$ const $_{i}=y_{i}$ for $i=1, \ldots, N$, using $(76)$.

By the second equation (75) and the boundary condition: $q_{S}=$ const $=0$ which implies $\theta_{S}(x) \equiv \theta_{\Gamma}$.

As the velocity $v_{S}$ is clearly zero by the boundary condition, the equation for the stress reads

$$
p\left(\eta_{S}, v_{S}, \theta_{S},\left\{Y_{i S}\right\}\right)=\sum_{i=1}^{N} R_{i} y_{i} \rho_{S}(x) \theta_{\Gamma}+\frac{a}{3} \theta_{\Gamma}^{4}=p_{\Gamma},
$$

which gives $\rho_{S}$, provided (77) is satisfied.

Let $\mathcal{N}>1$ be an arbitrarily large parameter and $K=K(\mathcal{N}), K_{i}=K_{i}(\mathcal{N}), i=$ $0,1, \ldots$, be positive nondecreasing functions of $\mathcal{N}$ which can depend also on the physical constants of the problem, and also on $\eta$ from (82) below but not directly on the initial data.

Let $\Psi(\zeta):=\zeta-\log \zeta-1$, for $\zeta>0$.

THEOREM 1. Suppose that the data are such that

$$
\begin{gathered}
\mathcal{N}^{-1} \leq \eta^{0} \leq \mathcal{N}, \quad\left\|v^{0}\right\|_{L^{2}(\Omega)}+\left\|\theta^{0}\right\|_{L^{4}(\Omega)}+\left\|\log \theta^{0}\right\|_{L^{1}(\Omega)} \leq \mathcal{N} \\
0 \leq Y_{i}^{0} \leq 1 \\
\left(1+\mathcal{N}^{-1}\right) \frac{a}{3} \theta_{\Gamma}^{4} \leq p_{\Gamma}
\end{gathered}
$$

Then:

1. The following bounds in $Q$ hold

$$
\begin{gathered}
0<\underline{\eta} \leq \eta(x, t) \leq \bar{\eta}=K \text { in } \bar{Q}, \\
\left\|\eta-\eta_{S}\right\|_{L^{2}(Q)}+\|v\|_{V_{2}(Q)}+\left\|\frac{v_{x}}{\sqrt{\theta}}\right\|_{L^{2}(Q)}+\left\|\sigma+p_{\Gamma}\right\|_{L^{2}(Q)} \leq K, \\
\|\theta\|_{L^{4, \infty}(Q)}+\|\log \theta\|_{L^{1, \infty}(Q)}+\left\|\frac{\sqrt{\kappa}}{\theta} \theta_{x}\right\|_{L^{2}(Q)} \leq K, \\
0 \leq Y_{i}(x, t) \leq 1 \quad \text { in } \bar{Q}, \\
\left\|\omega_{i}\left(\rho, \theta,\left\{Y_{j}\right\}_{j=1, \ldots, N}\right)\right\|_{L^{1}(Q)} \leq \mathcal{N}, \quad\left\|Y_{i x}\right\|_{L^{2}(Q)} \leq K .
\end{gathered}
$$

2. The following stabilization properties of the solution hold

$$
\begin{gathered}
\mathbf{V}^{(1)}(t):=\int_{\Omega}\left[\left(\eta(x, t)-\eta_{S}(x)\right)^{2}+v^{2}(x, t)\right. \\
\left.+(1+\theta(x, t))^{2}\left(\theta(x, t)-\theta_{\Gamma}\right)^{2}+\Psi\left(\frac{\theta(x, t)}{\theta_{\Gamma}}\right)\right] d x \rightarrow 0, \\
\left\|Y_{i}(\cdot, t)-Y_{i S}\right\|_{L^{2}(\Omega)} \rightarrow 0,
\end{gathered}
$$

as $t \rightarrow \infty$.

For completeness, let us state the corresponding global existence and uniqueness result. 
THEOREM 2. Under the hypotheses of Theorem 1 (recall that conditions (74) and $Y_{i}^{0} \in$ $L^{\infty}(\Omega)$ are supposed there), there exists a unique global solution to the problem (50)-(71) satisfying properties (73) along with $Y_{i} \in V_{2}\left(Q_{T}\right)$.

The proof may be adapted from [26]: we start from a local existence result, using our recent approach from [25] based mainly on the theory of $V_{2}\left(Q_{T}\right)$ and $H^{2,1}\left(Q_{T}\right)$ solutions of linear parabolic problems. The local solution appears simply as a fixed point of a specific nonlinear operator due to the Schauder fixed point theorem. Finally we use the previous a-priori estimates to get a global solution.

4.2. Sketch of the proof of Theorem 1. The proof mainly follows the strategy developed in [26] and relies on the behaviour of suitable Lyapunov functionals [22].

Let $\|\cdot\|_{G}=\|\cdot\|_{L^{2}(G)}$ and $z_{+}=\max \{z, 0\}$ for brevity. We shall use sometimes another notation for the compositions $b[\eta, \theta](x, t)=b(\eta(x, t), \theta(x, t))$, for $b=e, p, \kappa$, etc.

Suppose that the hypotheses of Theorem 1 are valid. By the definition and the conservation property (72) of species, one knows first that

$$
0 \leq Y_{i}(x, t) \leq 1 \text { in } Q .
$$

LEMMA 1. The following equality holds

$$
\frac{d}{d t}\left\langle Y_{i}\right\rangle+\left\langle\omega_{i}\right\rangle=0 \text { for } i=1, \ldots, N-1, \text { on } \mathbf{R}^{+} .
$$

Consequently the following bound in $Q$ and properties of the function $\left\langle Y_{i}\right\rangle$ hold

$$
\begin{gathered}
\left|\int_{Q} \omega_{i} d x d t\right|=\left\|\int_{\Omega} \omega_{i} d x\right\|_{L^{1}\left(\mathbf{R}^{+}\right)} \leq 2 M, \\
\left\langle Y_{i}(\cdot, t)\right\rangle \geq 0 \text { is nonincreasing for } 0 \leq i \leq N-1, \text { for } t \geq 0, \\
\left\langle Y_{N}(\cdot, t)\right\rangle \geq 0 \text { is nondecreasing, for } t \geq 0, \\
\left\langle Y_{i}(\cdot, t)\right\rangle \rightarrow Y_{i S} \text { for } i=1, \ldots, N, \text { as } t \rightarrow \infty,
\end{gathered}
$$

for some $0 \leq Y_{i S} \leq 1$.

Proof. Equality (89) follows from (53) and (70).

Using $\left.Y_{i}\right|_{t=0}=Y_{i}^{0}$, one gets

$$
\left\langle Y_{i}\right\rangle+\int_{0}^{t}\left\langle\omega_{i}(s)\right\rangle d s=\left\langle Y_{i}^{0}\right\rangle
$$

which implies bound (90) and all the properties of $\left\langle Y_{i}\right\rangle$, observing that $\frac{d}{d t}\left\langle Y_{i}\right\rangle \in L^{1}(0, T)$, for any $T<\infty$ and any $i=1, \ldots, N$.

LEMMA 2.

$$
\forall i \in(1, N): \quad \frac{1}{2}\left\|Y_{i}\right\|_{\Omega}^{2}+\int_{0}^{T} \int_{\Omega} \rho^{2} D_{i} Y_{i x}^{2} d x \leq K .
$$

Proof. For any $i \leq N-1$, multiplying (53) by $Y_{i}$, integrating by parts and using boundary conditions (70) and condition (62), we have 


$$
\frac{1}{2} \frac{d}{d t} \int_{\Omega} \sum_{i=1}^{N} Y_{i}^{2} d x+\int_{\Omega} \sum_{i=1}^{N} \rho^{2} D_{i} Y_{i x}^{2} d x \leq 0,
$$

which gives (92) after integrating on $(0, T)$.

Let us introduce the normalized specific volume and temperature $\tilde{\eta}:=\frac{\eta}{\eta_{0}}, \tilde{\theta}:=\frac{\theta}{\theta_{\Gamma}}$, where $\eta_{0}$ is a positive constant to be chosen latter, and let us denote by $\Psi_{r}$ the elementary function $\Psi_{r}(\zeta):=\zeta^{4}-\frac{4}{3} \zeta^{3}+\frac{1}{3} \geq 0$.

LEMma 3. The following inequality holds

$$
\begin{gathered}
\frac{d}{d t} \int_{\Omega}\left[\theta_{\Gamma}\left(\sum_{i=1}^{N} R_{i}\right) \Psi(\tilde{\eta})+\frac{1}{2} v^{2}+\left(\sum_{i=1}^{N} c_{V i}\right) \theta_{\Gamma} \Psi(\tilde{\theta})+\theta_{\Gamma} \sum_{i=1}^{N} R_{i} \Psi\left(Y_{i}\right)+a \eta \theta_{\Gamma}^{4} \Phi(\tilde{\theta})\right] d x \\
+\theta_{\Gamma} \int_{\Omega}\left(\frac{\nu \rho}{\theta} v_{x}^{2}+\frac{\kappa \rho}{\theta^{2}} \theta_{x}^{2}\right) d x \\
=\frac{d}{d t}\left(\theta_{\Gamma} \int_{\Omega} \sum_{i=1}^{N}\left[R_{i} Y_{i}+\mathcal{R} Y_{i}\left(\log \left(R_{i} Y_{i}\right)-1\right)\right] d x\right)+\theta_{\Gamma} \int_{\Omega} \frac{\theta_{x}}{\theta^{2}} \sum_{i=1}^{N} \rho_{i} V_{i} h_{i} d x
\end{gathered}
$$

Consequently the following bounds in $Q$ hold

$$
\begin{gathered}
\|\Psi(\tilde{\eta})\|_{L^{1, \infty}(Q)}+\|v\|_{L^{2, \infty}(Q)}+\|\Psi(\tilde{\theta})+\eta \Phi(\tilde{\theta})\|_{L^{1, \infty}(Q)} \\
+\sum_{i=1}^{N}\left\|Y_{i}\right\|_{L^{2, \infty}(Q)}+\sum_{i=1}^{N}\left\|Y_{i} \Psi\left(Y_{i}\right)\right\|_{L^{1, \infty}(Q)} \leq K, \\
\left\|\sqrt{\frac{\rho}{\theta}} v_{x}\right\|_{L^{2}(Q)}+\left\|\frac{\sqrt{\kappa \rho}}{\theta} \theta_{x}\right\|_{L^{2}(Q)}+\sum_{i=1}^{N}\left\|\rho \sqrt{D_{i}} Y_{i x}\right\|_{L^{2}(Q)} \leq K .
\end{gathered}
$$

Proof. From (51), (52) one gets

$$
\left(\frac{1}{2} v^{2}+e\right)_{t}=(\tau v-q)_{x}
$$

and using (64)

$$
\left(\frac{1}{2} v^{2}+e-\theta_{\Gamma} s\right)_{t}=\left[\tau v-\left(1-\frac{\theta_{\Gamma}}{\theta}\right) q-\frac{\theta_{\Gamma}}{\theta} \sum_{i=1}^{N} \rho_{i} V_{i} \mu_{i}\right]_{x}-\theta_{\Gamma} \varsigma_{S} .
$$

Integrating on $\Omega$ and using boundary conditions

$$
\frac{d}{d t} \int_{\Omega}\left(P_{\Gamma} \eta+\frac{1}{2} v^{2}+e-\theta_{\Gamma} s\right) d x+\theta_{\Gamma} \int_{\Omega} \varsigma_{S} d x=0
$$

Using the expression of $\varsigma_{S}$, we get

$$
\begin{aligned}
& \frac{d}{d t} \int_{\Omega}\left(p_{\Gamma} \eta+\frac{1}{2} v^{2}+e-\theta_{\Gamma} s\right) d x+\theta_{\Gamma} \int_{\Omega}\left(\kappa \rho \frac{\theta_{x}^{2}}{\theta^{2}}+\nu \rho \frac{v_{x}^{2}}{\theta}\right) d x \\
& \quad=\theta_{\Gamma} \int_{\Omega}\left(\frac{\theta_{x}}{\theta^{2}} \sum_{i=1}^{N} \rho_{i} h_{i} V_{i}+\sum_{i=1}^{N} \rho_{i} V_{i}\left(\frac{\mu_{i}}{\theta}\right)_{x}+\sum_{i=1}^{N} \frac{\mu_{i}}{\theta} \omega_{i}\right) d x
\end{aligned}
$$


Using the previously defined renormalized temperature and specific volume, the integrand in the left-hand side reads

$$
\mathcal{A}:=p_{\Gamma} \eta+\frac{1}{2} v^{2}+e-\theta_{\Gamma} s=\frac{1}{2} v^{2}+\mathcal{A}_{1}+\mathcal{A}_{2},
$$

with

$$
\mathcal{A}_{1}:=\left(p_{\Gamma}-\frac{1}{3} a \theta_{\Gamma}^{4}\right) \eta_{0} \tilde{\eta}-\theta_{\Gamma}\left(\sum_{i=1}^{N} R_{i}\right) \log \tilde{\eta},
$$

$$
\mathcal{A}_{2}:=-\theta_{\Gamma} \sum_{i=1}^{N} R_{i} \log Y_{i}+\theta_{\Gamma}\left(1+\log \theta_{\Gamma}\right) \sum_{i=1}^{N} c_{V i}-\theta_{\Gamma}\left(\sum_{i=1}^{N} c_{V i}\right) \Psi(\tilde{\eta})+a \eta \theta_{\Gamma}^{4} \Phi(\tilde{\theta}) .
$$

Choosing $\eta_{0}=\frac{\theta_{\Gamma} \sum_{i=1}^{N} R_{i}}{p_{\Gamma}-\frac{1}{3} a \theta_{\Gamma}^{4}}$, we obtain

$$
\mathcal{A}_{1}:=\theta_{\Gamma}\left(\sum_{i=1}^{N} R_{i}\right) \Psi(\tilde{\eta})+\theta_{\Gamma}\left(\sum_{i=1}^{N} R_{i}\right) .
$$

In the same stroke

$$
\begin{aligned}
\mathcal{A}_{2}:=\theta_{\Gamma} \sum_{i=1}^{N} R_{i} & \Psi\left(Y_{i}\right)-\theta_{\Gamma} \sum_{i=1}^{N} R_{i}\left(Y_{i}-1\right)+\theta_{\Gamma}\left(1+\log \theta_{\Gamma}\right) \sum_{i=1}^{N} c_{V i} \\
& +\theta_{\Gamma}\left(\sum_{i=1}^{N} c_{V i}\right) \Psi(\tilde{\eta})+a \eta \theta_{\Gamma}^{4} \Phi(\tilde{\theta})
\end{aligned}
$$

So we get

$$
\begin{gathered}
\frac{d}{d t} \int_{\Omega}\left(\theta_{\Gamma}\left(\sum_{i=1}^{N} R_{i}\right) \Psi(\tilde{\eta})+\frac{1}{2} v^{2}+\theta_{\Gamma} \sum_{i=1}^{N} R_{i} \Psi\left(Y_{i}\right)\right. \\
\left.+\theta_{\Gamma}\left(\sum_{i=1}^{N} c_{V i}\right) \Psi(\tilde{\theta})+a \eta \theta_{\Gamma}^{4} \Phi(\tilde{\theta})\right) d x \\
+\theta_{\Gamma} \int_{\Omega}\left(\kappa \frac{\theta_{x}^{2}}{\theta^{2}}+\frac{\nu}{\theta} \rho v_{x}^{2}\right) d x \\
=\frac{d}{d t}\left(\theta_{\Gamma} \int_{\Omega} \sum_{i=1}^{N} R_{i} Y_{i} d x\right)+\theta_{\Gamma} \int_{\Omega} \frac{\theta_{x}}{\theta^{2}} \sum_{i=1}^{N} \rho_{i} h_{i} V_{i} d x \\
+\theta_{\Gamma} \int_{\Omega}\left(\sum_{i=1}^{N} \rho_{i} V_{i}\left(\frac{\mu_{i}}{\theta}\right)_{x}+\sum_{i=1}^{N} \frac{\mu_{i}}{\theta} \omega_{i}\right) d x:=\mathcal{B}_{1}+\mathcal{B}_{2}+\mathcal{B}_{3} .
\end{gathered}
$$

Integrating by parts in the last term, we have

$$
\begin{gathered}
\mathcal{B}_{3}=\theta_{\Gamma} \int_{\Omega}\left(\sum_{i=1}^{N} \rho_{i} V_{i}\left(\frac{\mu_{i}}{\theta}\right)_{x}+\sum_{i=1}^{N} \frac{\mu_{i}}{\theta} \omega_{i}\right) d x \\
=-\theta_{\Gamma} \int_{\Omega} \sum_{i=1}^{N} D_{i} \rho^{2}\left(Y_{i}\right)_{x}\left(\frac{\mu_{i}}{\theta}\right)_{x} d x+\theta_{\Gamma} \int_{\Omega} \sum_{i=1}^{N} \frac{\mu_{i}}{\theta} \omega_{i} d x \\
=\theta_{\Gamma} \int_{\Omega} \sum_{i=1}^{N}\left(D_{i} \rho^{2}\left(Y_{i}\right)_{x}\right)_{x} \frac{\mu_{i}}{\theta} d x+\theta_{\Gamma} \int_{\Omega} \sum_{i=1}^{N} \frac{\mu_{i}}{\theta} \omega_{i} d x=\theta_{\Gamma} \int_{\Omega} \sum_{i=1}^{N}\left(Y_{i}\right)_{t} \frac{\mu_{i}}{\theta} d x .
\end{gathered}
$$


As the chemical potential for perfect gas is $\mu_{i}=\mu_{0}+\mathcal{R} \theta \log \frac{p_{i}}{p_{0}}$, where $\mu_{0}$ and $p_{0}$ are reference constants and $\mathcal{R}$ is the perfect gas constant, we compute

$$
\frac{\mu_{i}}{\theta}=\frac{\mu_{0}}{\theta}+\mathcal{R} \log R_{i}+\mathcal{R} \log \rho+\mathcal{R} \log \theta+\mathcal{R} \log Y_{i}+\mathcal{R} \log p_{0},
$$

using the conservation of species, we conclude that

$$
\mathcal{B}_{3}=\theta_{\Gamma} \mathcal{R} \int_{\Omega} \sum_{i=1}^{N}\left(Y_{i}\right)_{t}\left(\log R_{i}+\log Y_{i}\right) d x .
$$

Finally

$$
\mathcal{B}_{1}+\mathcal{B}_{3}=\frac{d}{d t}\left(\theta_{\Gamma} \int_{\Omega} \sum_{i=1}^{N}\left[R_{i} Y_{i}+\mathcal{R} Y_{i}\left(\log \left(R_{i} Y_{i}\right)-1\right)\right] d x\right)
$$

Plugging (97) into (96), we get (93).

To get the bounds (94) and (94) we integrate (93) on $(0, T)$, for any $T>0$

$$
\begin{gathered}
\int_{\Omega}\left[\theta_{\Gamma} \sum_{i=1}^{N} R_{i} \Psi(\tilde{\eta})+\right. \\
\left.+\frac{1}{2} v^{2}+\sum_{i=1}^{N} c_{V i} \theta_{\Gamma} \Psi(\tilde{\theta})+\theta_{\Gamma} \sum_{i=1}^{N} R_{i} \Psi\left(\tilde{Y}_{i}\right)+a \eta \theta_{\Gamma}^{4} \Phi(\tilde{\theta})\right] d x \\
+\theta_{\Gamma} \int_{0}^{T} \int_{\Omega}\left(\frac{\nu \rho}{\theta} v_{x}^{2}+\frac{\kappa \rho}{\theta^{2}} \theta_{x}^{2}\right) d x d t \\
=\theta_{\Gamma} \int_{\Omega} \sum_{i=1}^{N}\left[R_{i} Y_{i}+\mathcal{R} Y_{i}\left(\log \left(R_{i} Y_{i}\right)-1\right)\right] d x+\theta_{\Gamma} \int_{0}^{T} \int_{\Omega} \frac{\theta_{x}}{\theta} \sum_{i=1}^{N} \rho_{i} h_{i} V_{i} d x d t \\
+\int_{\Omega}\left[\theta_{\Gamma} \sum_{i=1}^{N} R_{i} \Psi\left(\tilde{\eta^{0}}\right)+\frac{1}{2} v^{0^{2}}+\sum_{i=1}^{N} c_{V i} \theta_{\Gamma} \Psi\left(\tilde{\theta}^{0}\right)+\theta_{\Gamma} \sum_{i=1}^{N} R_{i} \Psi\left(\tilde{Y}_{i}^{0}\right)+a \eta^{0} \theta_{\Gamma}^{4} \Phi\left(\tilde{\theta}^{0}\right)\right. \\
\left.-\theta_{\Gamma} \sum_{i=1}^{N}\left[R_{i} Y_{i}^{0}+\mathcal{R} Y_{i}^{0}\left(\log \left(R_{i} Y_{i}^{0}\right)-1\right)\right]\right] d x
\end{gathered}
$$

We can bound $\mathcal{B}_{2}$ (the second contribution in (98)) by using the Cauchy-Schwarz inequality and (92)

$$
\begin{gathered}
\left|\mathcal{B}_{2}\right| \leq \theta_{\Gamma} \int_{\Omega} \frac{\left|\theta_{x}\right|}{\theta} \sum_{i=1}^{N} R_{i} D_{i} c_{P i} \rho\left|\left(Y_{i}\right)_{x}\right| d x \\
\leq \frac{\epsilon}{2} \theta_{\Gamma} \int_{\Omega} \kappa \rho \frac{\theta_{x}^{2}}{\theta^{2}} d x+\frac{1}{2 \epsilon} \theta_{\Gamma} \int_{\Omega} \frac{\rho}{\kappa}\left(\sum_{i=1}^{N} R_{i} D_{i} c_{P i} \rho\left(Y_{i}\right)_{x}\right)^{2} d x \\
\leq \frac{\epsilon}{2} \theta_{\Gamma} \int_{\Omega} \kappa \rho \frac{\theta_{x}^{2}}{\theta^{2}} d x+\frac{\delta}{2 \epsilon} \theta_{\Gamma} \int_{\Omega} \sum_{i=1}^{N} D_{i} \rho^{2}\left(Y_{i x}\right)^{2} d x,
\end{gathered}
$$

where $\delta=\kappa_{1}^{-1} \max _{i}\left\{R_{i}^{2} D_{i} c_{P}^{2}\right\}$, which gives the estimate

$$
\left|\mathcal{B}_{2}\right| \leq \frac{\epsilon}{2} \theta_{\Gamma} \int_{\Omega} \kappa \rho \frac{\theta_{x}^{2}}{\theta^{2}} d x+K_{1}(\mathcal{N}),
$$

where $\epsilon>0$. 
Finally, using lemma 2 and (98) and for $\epsilon$ small enough, we get the inequality

$$
\begin{gathered}
\int_{\Omega}\left[\theta_{\Gamma} \sum_{i=1}^{N} R_{i} \Psi(\tilde{\eta})+\frac{1}{2} v^{2}+\frac{1}{2} \sum_{i=1}^{N} Y_{i}^{2}+\sum_{i=1}^{N} c_{V i} \theta_{\Gamma} \Psi(\tilde{\theta})+\theta_{\Gamma} \sum_{i=1}^{N} R_{i} \Psi\left(\tilde{Y}_{i}\right)+a \eta \theta_{\Gamma}^{4} \Phi(\tilde{\theta})\right] d x \\
+\theta_{\Gamma} \int_{0}^{T} \int_{\Omega}\left(\frac{\nu \rho}{\theta} v_{x}^{2}+\frac{\kappa \rho}{\theta^{2}} \theta_{x}^{2}+\sum_{i=1}^{N} \rho^{2} D_{i}\left(Y_{i x}\right)^{2}\right) d x d t \leq K_{2}(\mathcal{N}),
\end{gathered}
$$

from which all the estimates (94) and (94) follow.

From the previous estimates one gets the following bounds in $Q$ for $\eta$ and $\theta$

$$
\|\eta\|_{L^{1, \infty}(Q)} \leq K, \quad\|\theta\|_{L^{1, \infty}(Q)}+\|\log \theta\|_{L^{1, \infty}(Q)}+\left\|\eta \theta^{4}\right\|_{L^{1, \infty}(Q)} \leq K .
$$

Now, by [26], the following implicit formula for the specific volume $\eta$ holds

$$
\eta=\frac{1}{E^{(\delta)}}\left[\eta^{0}+\frac{1}{\nu} I_{0}\left(E^{(\delta)} \eta(p-\delta)\right)\right] \text { in } \bar{Q},
$$

with a parameter $\delta$, where

$$
E^{(\delta)}(x, t):=B(x, t) e^{\frac{1}{\nu}\left(p_{\Gamma}(x)-\delta\right) t} \text {, with } K^{-1} \leq B:=e^{\frac{1}{\nu} I^{*}\left(v-v^{0}\right)} \leq K \text { in } \bar{Q} .
$$

Using this formula together with the energy bounds (100)(104) and (100)(104), we obtain the crucial density bounds

$$
0<\underline{\eta} \leq \eta(x, t) \leq \bar{\eta} \text { in } \bar{Q} .
$$

Then one checks the additional $L^{p}$ estimates

$$
\begin{gathered}
\|\tilde{\theta}-1\|_{C(\bar{\Omega})}+\left\|\tilde{\theta}^{2}-1\right\|_{C(\bar{\Omega})}+\left\|\sqrt{\eta}\left(\tilde{\theta}^{4}-1\right)\right\|_{L^{2}(\Omega)} \leq K A, \\
\|\Psi(\tilde{\theta})\|_{C(\bar{\Omega})}+\left\|\eta \Psi_{r}(\tilde{\theta})\right\|_{L^{1}(\Omega)} \leq K A^{2},
\end{gathered}
$$

with $A:=\left\|\frac{\sqrt{\rho}}{\theta} \theta_{x}\right\|_{L^{2}(\Omega)}$ satisfying $\|A\|_{L^{2}\left(\mathbf{R}^{+}\right)} \leq K$.

Using (102), the following inequalities hold for $i=1, \ldots, N$

$$
\frac{1}{2} \frac{d}{d t}\left\|Y_{i}-\left\langle Y_{i}\right\rangle\right\|_{\Omega}^{2}+D_{i} \underline{\rho}^{2}\left\|\left(Y_{i}\right)_{x}\right\|_{\Omega}^{2} \leq \int_{\Omega} Y_{i} \omega_{i} d x \text { on } \mathbf{R}^{+} .
$$

Consequently the second bound (86) follows. Moreover $\frac{d}{d t} \int_{\Omega}\left(Y_{i}-\left\langle Y_{i}\right\rangle\right)^{2} d x \in L^{1}(0, T)$, for any $T<\infty$ and the stabilization property (88) holds.

In the spirit of [26] we introduce now the functionals

$$
\begin{gathered}
\mathcal{V}^{(0)}:=\int_{\Omega}\left\{\theta_{\Gamma}\left[\left(\sum_{i=1}^{N} R_{i} \Psi\left(\tilde{\eta}_{i}\right)\right)+\beta_{0} \nu \eta_{S} \Psi(\tilde{\eta})\right]+\frac{1}{2} v^{2}+\beta_{0} v I\left(\eta-\eta_{S}\right)\right. \\
\left.+\theta_{\Gamma}\left(\sum_{i=1}^{N} c_{V i} Y_{i}\right) \Psi(\tilde{\theta})+a \theta_{\Gamma}^{4} \eta \Psi_{r}(\tilde{\theta})\right\} d x, \\
\mathcal{Y}^{(0)}:=\int_{\Omega}\left[\beta_{0} \sum_{i=1}^{N} R_{i} \rho \rho_{S} Y_{i}\left(\eta-\eta_{S}\right)^{2}+\frac{\nu}{\tilde{\theta}} \rho v_{x}^{2}-\beta_{0} v^{2}+\theta_{\Gamma} \frac{\rho \kappa}{\theta^{2}} \theta_{x}^{2}\right] d x, \\
\mathbf{Y}^{(0)}:=\int_{\Omega}\left[\left(\eta-\eta_{S}\right)^{2}+\frac{v_{x}^{2}}{\theta}+\frac{\kappa}{\theta^{2}} \theta_{x}^{2}\right] d x,
\end{gathered}
$$

with a parameter $\beta_{0}$. 
Then, reasoning similarly to [26], the following inequality holds

$$
\frac{d \mathcal{V}^{(0)}}{d t}+\mathcal{Y}^{(0)} \leq \beta_{0} \int_{\Omega}\left[p\left(\eta, \theta ;\left\{Y_{i}\right\}_{i=1, \ldots, N}\right)-p\left(\eta, \theta_{\Gamma} ;\left\{Y_{i S}\right\}_{i=1, \ldots, N}\right)\right]\left(\eta-\eta_{S}\right) d x
$$

together with the estimates, for a fixed and sufficiently small $\beta_{0}:=\frac{1}{K}$,

$$
\begin{gathered}
\frac{d \mathcal{V}^{(0)}}{d t}+K^{-1} \mathbf{Y}^{(0)} \leq \int_{\Omega}\left[p\left(\eta, \theta ;\left\{Y_{i}\right\}_{i=1, \ldots, N}\right)-p\left(\eta, \theta_{\Gamma} ;\left\{Y_{i S}\right\}_{i=1, \ldots, N}\right)\right]\left(\eta-\eta_{S}\right) d x \\
K^{-1} \mathbf{V}^{(1)} \leq \mathcal{V}^{(0)} \leq K \mathbf{V}^{(1)} \leq K_{1} \mathbf{Y}^{(0)}
\end{gathered}
$$

where $\mathbf{V}^{(1)}$ has been introduced in $(87)$.

In fact, due to $\|\theta\|_{L^{1}(\Omega)} \leq K$ (see $\left.(100)\right)$, we have

$$
\int_{\Omega} v^{2} d x \leq M\left\|v_{x}\right\|_{L^{1}(\Omega)}^{2} \leq M\|\theta\|_{L^{1}(\Omega)}\left\|\frac{v_{x}}{\sqrt{\theta}}\right\|_{\Omega}^{2} \leq M K\left\|\frac{v_{x}}{\sqrt{\theta}}\right\|_{\Omega}^{2} .
$$

Finally, using the uniform bounds $\underline{\rho} \leq \rho \leq \bar{\rho}$ and choosing $\varepsilon:=\frac{1}{K}$ and then $\beta_{0}:=\frac{1}{K_{1}}$, both small enough, we obtain (107). Taking into account inequality (104) also, we obtain the last inequality (108) as well. Consequently the stabilization property (87) is valid, which ends the proof of the theorem

\section{References}

[1] S. N. Antontsev, A. V. Kazhikhov and V. N. Monakhov, Boundary Value Problems in Mechanics of Nonhomogeneous Fluids, North-Holland, Amsterdam, 1990.

[2] M. Arnould and M. Rayet, Nuclear reactions in astrophysics, Ann. Phys. Fr. 15 (1990), $183-254$.

[3] J. Bebernes and A. Bressan, Global a-priori estimates for a viscous reactive gas, Proc. Roy. Soc. Edinburgh 101A (1985), 321-333.

[4] J. Bebernes and D. Eberly, Mathematical Problems from Combustion Theory, Springer, Berlin, 1989.

[5] A. Bermúdez de Castro, Continuum Thermomechanics, Birkhäuser, Basel, 2005.

[6] M. Bisi, M. Groppi and G. Spiga, Fluid-dynamic equations for reacting gas mixtures, Applications of Mathematics 50 (2005), 43-62.

[7] M. Bisi and G. Spiga, Hydrodynamical limit for a gas with chemical reactions, in: "WASCOM 2003" 13th Conference on Waves and Stability in Continuous Media, R. Monaco, et al. (eds.) World Scientific, Singapore, 2004.

[8] A. Bressan, Global solution for the one-dimensional equations of a viscous reactive gas, Boll. U.M.I. 5B (1986), 291-308.

[9] R. Brun, Introduction à la dynamique des gaz réactifs, Cépaduès Editions, 2005.

[10] J. M. Burgers, Flow Equations for Composite Gas, Academic Press, 1969.

[11] S. Chapman and T. G. Cowling, The Mathematical Theory of Non-uniform Gas, Cambridge Math. Library, 1999.

[12] G.-Q. Chen, Global solution to the compressible Navier-Stokes equations for a reacting mixture, SIAM J. Math. Anal. 23 (1992), 609-634.

[13] G.-Q. Chen, D. Hoff and K. Trivisa, On the Navier-Stokes equations for exothermically reacting compressible fluids, Acta Math. Appl. Sinica 18 (2002), 15-36. 
[14] G.-Q. Chen, D. Hoff and K. Trivisa, Global solution to a model for exothermically reacting compressible flows with large discontinuous data, Arch. Rat. Mech. Anal. 166 (2003), 321358.

[15] B. Desjardins, Regularity results for two-dimensional flows of multiphase viscous fluids, Arch. Rational Mech. Anal. 137 (1997), 135-158.

[16] D. Donatelli and K. Trivisa, On the motion of a viscous compressible radiative reacting gas, Commun. in Math. Physics 265 (2006), 463-491.

[17] D. A. Drew and S. L. Passman, Theory of Multicomponent Fluids, Springer, Berlin, 1999.

[18] B. Ducomet, Hydrodynamical models of gaseous stars, Reviews of Math. Phys. 8 (1996), 957-1000.

[19] B. Ducomet, Some asymptotics for a reactive Navier-Stokes-Poisson system, Math. Methods Models Appl. Sci. 9 (1999), 1039-1076.1569

[20] B. Ducomet, A model of thermal dissipation for a one-dimensional viscous reactive and radiative gas, Math. Meth. Appl. Sci. 22 (1999), 1323-1349.

[21] B. Ducomet, Stability problems in the dynamics of gaseous stars, in: Function Spaces, Differential Operators and Nonlinear Analysis ( Milovy, 2004), P. Drábek and J. Rákoznik (eds.), Mathematical Institute AS CR, Prague, 2005, 72-86.

[22] B. Ducomet, On a 1D multicomponent model in stellar evolution, in preparation.

[23] B. Ducomet and E. Feireisl, A regularizing effect of radiation in the equations of fluid dynamics, Mathematical Methods in the Applied Sciences 28 (2005), 661-685.

[24] B. Ducomet and E. Feireisl, On the dynamics of gaseous stars, Arch. Rational Mechanics and Analysis 177 (2005), 185-229.

[25] B. Ducomet and A. A. Zlotnik, Stabilization for equations of a one-dimensional viscous compressible heat-conducting media with non-monotone equation of state, J. Diff. Equations 194 (2003), 51-81.

[26] B. Ducomet and A. Zlotnik, Lyapunov functional method for $1 D$ radiative and reactive viscous gas dynamics, Arch. Rational Mechanics and Analysis 177 (2005), 185-229.

[27] B. Ducomet and A. Zlotnik, On the large-time behavior of $1 D$ radiative and reactive viscous flows for higher-order kinetics, Nonlinear Analysis 63 (2005), 1011-1033.

[28] E. Feireisl, Dynamics of Viscous Compressible Fluids, Oxford University Press, Oxford, 2004.

[29] E. Feireisl and A. Novotný, On a simple model of reacting compressible flows arising in astrophysics, Proceedings of the Royal Soc. of Edinburgh 135A (2005), 1169-1194.

[30] M. Forestini, Principes fondamentaux de structure stellaire, Gordon and Breach, Overseas Publishers Association, 1999.

[31] J. Frehse, S. Goj and J. Malek, Existence of solutions to a Stokes-like system for mixtures, SIAM Journal on Mathematical Analysis 36 (2005), 1259-1281.

[32] J. Frehse, S. Goj and J. Malek, A uniqueness result for a model for mixtures in the absence of external forces and interaction momenta, preprint.

[33] G. Gallavotti, Foundations of Fluid Dynamics, Springer, Berlin, 2002.

[34] V. Giovangigli, Multicomponent Flow Modelling, Birkhäuser, Basel, 1999.

[35] B. Guo and P. Zhu, Asymptotic behaviour of the solution to the system for a viscous reactive gas, J. Diff. Equations 155 (1999), 177-202.

[36] M. Ishii, Thermo-Fluid Dynamic Theory of Two-Phase Flow, Editions Eyrolles, 1975.

[37] A. V. Kazhikov and A. N. Petrov, Well-posedness of the initial-boundary value problem for a model system of a multicomponent mixture, Dinam. Splosh. Sredy 174 (1978), 61-73.

[38] R. Kippenhahn and A. Weigert, Stellar Structure and Evolution, Springer, Berlin, 1994. 
[39] N. Kolev, Multiphase Flow Dynamics, Vol. I and II, Springer, Berlin, 2002.

[40] L. Landau and E. Lifchitz, Mécanique des fluides, Editions Mir, Moscou, 1971.

[41] O. A. Ladyženskaja, V. A. Solonnikov and N. N. Ural'ceva, Linear and Quasilinear Equations of Parabolic Type, AMS, Providence, 1968.

[42] J. Lequeux, E. Falgarone and C. Ryter, The Interstellar Medium, Springer, Berlin, 2003.

[43] P. L. Lions, Mathematical Topics in Fluid Mechanics, Vol. 2, Compressible Models, Oxford Science Publication, Oxford, 1998.

[44] M. Ya. Marov and A. V. Kolesnichenko, Mechanics of Turbulence of Multicomponent Gas, Kluwer, 2001.

[45] D. M. Mihalas and B. Weibel-Mihalas, Foundations of Radiation Hydrodynamics, Oxford University Press, New York, 1984.

[46] A. Nouri and F. Poupaud, An existence theorem for the multifluid Navier-Stokes problem, J. Diff. Eq. 122 (1995), 71-88.

[47] S. F. Shandarin and Ya. B. Zeldovitch, The large-scale structure of the universe: turbulence, intermittency, structures in a self-gravitating medium, Rev. Modern Phys. 61 (1989), 185-220.

[48] I. Straškraba and A. A. Zlotnik, On a decay rate for 1D-viscous compressible barotropic fluid equations, J. Evolution Equations 2 (2002), 69-96.

[49] M. Tabata, Local solutions to the Cauchy problem for the system of equations describing the motion of mixture of compressible viscous and heat-conductive fluids, Kobe J. Math. 9 (1992), 195-205.

[50] N. Tanaka, Global existence of two phase non-homogeneous viscous incompressible fluid flow, Comm. Partial Diff. Eq. 18 (1993), 41-81.

[51] F. Williams, Combustion Theory, Benjamin/Cummings, Menlo Park, 1985.

[52] S. Yanagi, Asymptotic stability of the solutions to a full one-dimensional system of heatconducting reactive compressible viscous gas, Jap. J. Indust. Appl. Math. 15 (1998), 423442.

[53] A. A. Zlotnik, Uniform estimates and stabilization of the solutions of a system of equations of the one-dimensional motion of a multicomponent barotropic mixture, Mat. Zametki 58 (1995), 307-312 (in Russian).

[54] A. A. Zlotnik and S. N. Puzanov, Correctness of the problem of viscous gas burning in the case of nonsmooth data and a semidiscrete method of its solution, Math. Notes 65 (1999), 793-797. 